\title{
Consideraciones para el aprendizaje autónomo en el entorno asíncrono: caso de implementación gradual de aula invertida en ingeniería
}

\author{
Teth Azrael Cortes Aguilar ${ }^{\mathrm{a}}$,Sofía Estelles-Miguel ${ }^{\mathrm{b}}$ \\ ${ }^{a}$ Instituto Tecnológico José Mario Molina Pasquel y Henríquez, Mexico, ${ }^{b}$ Departamento de \\ Organización de Empresas, Universitat Politècnica de València, España
}

\begin{abstract}
Resumen
El presente artículo aborda la implementación gradual en educación superior del modelo de aula invertida para el caso de estudiantes de primer semestre de ingeniería. La transición de clase tradicional hacia aula invertida inició en el entorno síncrono, dentro del aula, con la integración del aprendizaje activo basado en problemas, reportando resultados favorables. Sin embargo, los resultados muestran una disminución en el rendimiento relacionado con un mayor uso de TIC en el entorno asíncrono. En consecuencia, para lograr el aprendizaje autónomo, se propone considerar en la etapa de diseño del aula invertida, el estilo de aprendizaje, la disponibilidad de recursos tecnológicos y las competencias digitales de los estudiantes.
\end{abstract}

Palabras clave: aula invertida, aprendizaje activo, educación en ingeniería.

\section{Introducción}

La definición más sencilla de aula invertida es dada por Lage et al. (2000) "Invertir el aula significa que los eventos que tradicionalmente han tenido lugar dentro del aula ahora tienen lugar fuera del aula y viceversa". Esta definición implicaría que el aula invertida simplemente representa un reordenamiento de las actividades dentro y fuera del aula, sin embargo, en la práctica este no es el caso (Demetry, 2010). De acuerdo a Staker y Horn (2012) el aula invertida es un programa de educación formal clasificado como un submodelo en el aprendizaje híbrido en el cual a los estudiantes se les permite elegir el lugar donde reciben el contenido, la instrucción y controlar el ritmo de actividades a través de los recursos en línea. La mayor parte de las investigaciones sobre aula invertida emplea actividades de aprendizaje interactivo en grupo, sustentadas en las teorías de aprendizaje centradas en el alumno, basadas en las obras de Piaget de 1967 y Vygotsky et al. (1980). El 
Consideraciones para el aprendizaje autónomo en el entorno asíncrono: caso de implementación gradual de aula invertida en ingeniería.

constructivismo se considera la fuente para las teorías del aprendizaje activo basado en problemas ABP (Grabinger y Dunlap, 1995). Bishop y Verlerger (2013) aportan una definición de aula invertida más apropiada, como una técnica educativa que consta de dos partes, actividades interactivas de aprendizaje grupal dentro del aula e instrucción individual directa basada en las tecnológicas de la Información y comunicación fuera del aula.

En el marco de referencia 2018 del Consejo de Acreditación de la Enseñanza de la Ingeniería A.C. se establece en el apartado 3.4 sobre atributos del perfil de egreso y objetivos educacionales que el egresado debe ser capaz de comunicarse efectivamente con diferentes audiencias; formular y resolver problemas de ingeniería; trabajar efectivamente en equipos que establecen metas, planean tareas, cumplen fechas límite y analizan riesgos e incertidumbre; también debe ser capaz de aplicar, analizar y sintetizar procesos de diseño de ingeniería que resulten en proyectos (Consejo de Acreditación de la Enseñanza de la Ingeniería A.C., 2017). Varios de estos criterios son difíciles de enseñar y evaluar efectivamente con conferencias informativas o exámenes de preguntas cerradas. Sin embargo, el método de aprendizaje basado en problemas $\mathrm{ABP}$, puede ser mucho más efectivo para lograr estos objetivos, pero la aplicación del ABP se ve obstaculizada por el hecho de que el plan de estudios para los programas de ingeniería ya está muy saturado de temas y en este tipo de programas parecer imposible su integración. Por el contrario, asignarles a los estudiantes como tarea, ver conferencias grabadas deja tiempo libre en clase para realizar actividades interactivas que no pueden ser automatizadas mediante las TIC, este es el concepto clave del aula invertida (Bishop y Verleger, 2013 y Oltra et al, 2017).

Aunque la tecnología no define el aula invertida, Bergmann y Sams (2012) mencionan una serie de beneficios cuando los materiales están disponibles en un formato combinado, en línea y en clase. Sin embargo Dahlstrom et al. (2015) advierten que los estudiantes que no sean capaces de trabajar con materiales de aprendizaje en línea sin ayuda, pueden requerir orientación del docente. Además los estudiantes que no están seguros acerca de cómo lograr un aprendizaje autónomo exitoso, sienten la necesidad de retroalimentación inmediata y pueden encontrar desafiante el material en línea asincrónico, hasta el punto de llegar a necesitar capacitación en estrategias centradas en el alumno.

La expansión del modelo de aula invertida, pese a tener una amplia aceptación en escenarios estadounidenses, Martínez et al. (2014) señalan que de diez artículos revisados, el 50\% reporta una mejora en la adquisición de conocimiento al comparar el aula invertida y el aula tradicional, lo cual no presenta un incremento significativo en el rendimiento escolar, si no que las ventajas se dan en términos de mejoría en la percepción del aprendizaje, así como un mayor grado de satisfacción de los estudiantes al involucrarse con dicha experiencia educativa, también refieren una mejoría en cuanto a mayor cantidad de contenido abarcado y menor esfuerzo para resolver las actividades. Suponen que los 
principios del aula invertida no han sido correctamente aplicados o que se requieren periodos de uso mayores para poder evaluar su impacto en todas las partes implicadas.

El papel de los estudiantes en el aula invertida es usar métodos de aprendizaje autónomos para revisar y considerar críticamente materiales fuera de clase y luego aplicar activamente en clase lo aprendido en un ambiente colaborativo. Lo anterior, es un marco útil para determinar qué habilidades de pensamiento se deben enseñar en línea y cuales se deben enseñar de manera presencial. De acuerdo a la taxonomía de Bloom, el docente podría razonablemente esperar que los estudiantes sean capaces de aprender, recordar y comprender un tema en un nivel básico en línea; y entonces, utilizar habilidades de pensamiento de orden superior para aplicar, analizar, evaluar y crear nuevo material en el aula sincrónica, ver Figura 1. Estes et al. (2014) recomiendan que los estudiantes más capaces se conviertan en tutores pares para sus compañeros dentro del aula, ayudando con las operaciones básicas que aún pueden requerir de atención durante el tiempo de clase.

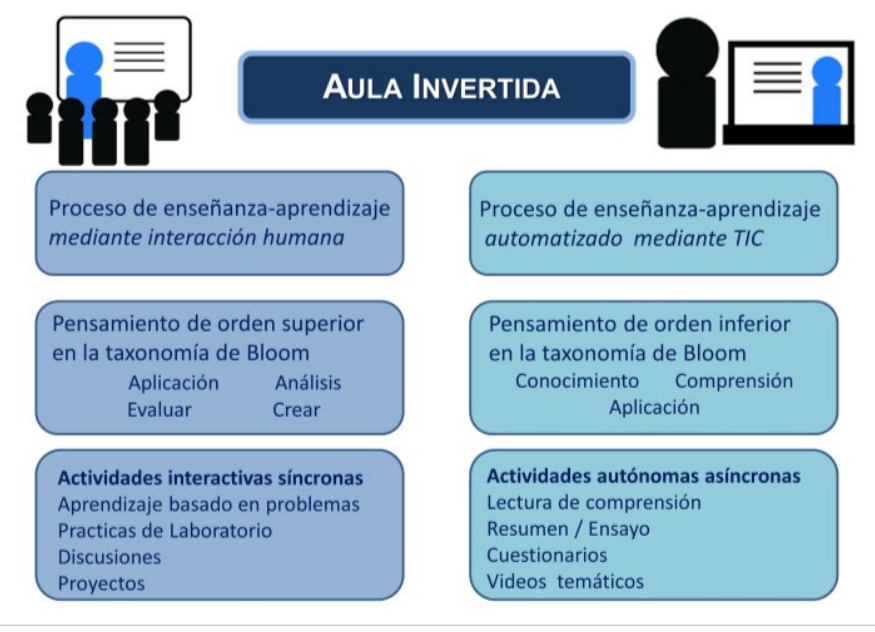

Fig. 1 Descripción del aula invertida por componentes sincrono y asíncrono.

En un enfoque tradicional (Francl, 2014), los profesores han conducido sus clases en la siguiente secuencia de eventos:

1. Presentación de temas a través de diapositivas de PowerPoint, videos y otros medios visuales y auditivos en clase.

2. Preguntas y respuestas durante o después de la conferencia.

3. Ejercicios prácticos o tarea para los estudiantes fuera de clase.

4. Después de la entrega de la tarea, explicación de las respuestas correctas.

5. Realización de un examen para valorar la asimilación del tema.

En contraste con el enfoque tradicional, Francl (2014) propone la siguiente secuencia de actividades para que se lleve a cabo el aprendizaje invertido. 
Consideraciones para el aprendizaje autónomo en el entorno asíncrono: caso de implementación gradual de aula invertida en ingeniería.

1. Automatizar las presentaciones de temas, facilitando la autorregulación.

2. Adquirir material complementario de fuentes distintas al libro de referencia.

3. Facilitar la visualización de contenido y material a los estudiantes para cualquier dispositivo, en cualquier lugar y en cualquier momento.

4. Solicitar a los estudiantes que consulten material asíncrono de tipo conferencias fuera del aula.

5. Proporcionar un cuestionario corto en línea para la autoevaluación del aprendizaje.

6. Permitir a los estudiantes volver a tomar ese cuestionario, por lo tanto, utilizarlo como una experiencia de aprendizaje formativa.

7. Permitir que los estudiantes completen actividades en el aula, ya sea individualmente o en grupos.

8. Llevar a cabo un foro abierto en clase para discutir sobre el tema y presentar una explicación más detallada de temas complejos según sea necesario.

9. Aplicar un examen formal para evaluar la comprensión del tema.

El presente trabajo se sustenta en la revisión de los autores Estes, Ingram y Liu (2014) del aula invertida, donde proponen un modelo simple de implementación en tres etapas.

Pre-Class. En lugar de utilizar el tiempo de clase presencial para informar, comunicar y generar conciencia, el instructor mueve este tipo de actividades a un entorno asíncrono disponible en línea. Las instrucciones para procedimientos que cambian muy poco con el tiempo son las más apropiadas para este tipo de entorno.

In-Class. En el aula, el docente usa indicaciones o el método socrático a través de preguntas para aumentar la interacción con el alumno mediante discusiones en clase, observación o el uso de tecnología. La colaboración entre los estudiantes también es importante, y es común usar la retroalimentación de los compañeros y la instrucción entre pares para el descubrimiento y la práctica.

Post-Class. Se establece en los momentos anterior y posterior de que se hayan elaborado los materiales asíncronos y síncronos de la conversión hacia el aula invertida, donde el profesor tiene la oportunidad de aumentar y mantener la motivación de los estudiantes para participar fuera del tiempo de clase y evaluar el progreso del alumno. El papel del docente se traslada de un instructor a la de un entrenador del aprendizaje que se apoya en el desarrollo de habilidades de autorregulación de los estudiantes, necesarios para el éxito en el entorno invertido.

Cabe distinguir, que para Flipped Learning Network (2014) los términos de aula invertida y aprendizaje invertido no son intercambiables y el primero no siempre puede conducir al segundo. En el aula invertida las actividades grupales dentro del salón se mueven hacia el espacio individual y las actividades individuales se mueven hacia el espacio grupal. En el 
aprendizaje invertido la instrucción directa se mueve del espacio de aprendizaje grupal al espacio de aprendizaje individual y el espacio grupal se transforma en un entorno dinámico e interactivo en el que el profesor guía a sus estudiantes para aplicar conceptos y participar creativamente. En la encuesta de la Red de Aprendizaje Sophia \& Flipped, 2014, (Flipped Learning Network, 2014) nueve de cada diez docentes reportaron mejoras en el rendimiento de sus estudiantes. Sin embargo, también señalan retos para su implementación, como la adquisición de conocimientos fundamentales en el entorno asincrónico, donde para tener éxito, los estudiantes deben reconocer y demostrar habilidades de aprendizaje autónomo. En las sesiones presenciales de aula invertida los docentes deben ser capaces de responder a preguntas espontáneas de sus estudiantes. También se deben considerar problemas en la creación de materiales en línea y acceso a la tecnología.

Para investigar el impacto del aula invertida sobre el aprendizaje de estudiantes de educación superior, Zhao y Ho (2014) de la Universidad de Harvard reportan el empleo de un diseño cuasi-experimental que recabó y comparó las calificaciones de exámenes de medio periodo en 2011 y 2013. En la opinión de los estudiantes un 46\% prefirió un modelo invertido y un $38 \%$ prefirió el modelo tradicional. También reportan que no existe una evidencia de impacto significativo en las calificaciones de medio periodo. El modelo de aula invertida se apoyó en la plataforma edX de aprendizaje abierto en línea, desarrollado en colaboración con el Massachusetts Institute of Technology MIT, disponible desde 2012. En el presente trabajo de implementación gradual del aula invertida se reportan las calificaciones de los ciclos 2012B a 2016B. No se realizaron encuestas de opinión entre los estudiantes porque es no es un objetivo la comparación entre el modelo tradicional y el modelo invertido, ampliamente discutido en investigaciones anteriores, Zhao y Ho (2014), Francl (2014) y Martínez et al. (2014).

La teoría del aprendizaje es considerada como parte esencial en el enfoque del aprendizaje centrado en el alumno. El estilo individual de aprendizaje y su relación con el aprendizaje en línea son importantes para mejorar el rendimiento de los estudiantes en los modelos de aprendizaje activos, especialmente para el estilo de aprendizaje visual del modelo VAK (Pekic et al., 2014). Los estudiantes tienen fortalezas y debilidades concretas que pueden aprovecharse o mejorarse mediante la instrucción. El aprendizaje basado en proyectos con tecnología permite que los estudiantes aprendan a identificar como aprovechar las fortalezas de sus estilos de aprendizaje, ayudándolos a desarrollar su pensamiento y convertirlos en aprendices más independientes (Intel Teach Program, 2012). En el modelo VAK propuesto por Neil Fleming, los estudiantes son identificados de acuerdo a su preferencia para aprender mejor con la ayuda de señales visuales, auditivas, kinestésicas o escritas (Muthyala, 2014). 
Los aprendices visuales son personas que aprenden mejor observando imágenes o vídeos.

Los aprendices auditivos son personas que prefieren aprender escuchando una conferencia o una discusión. Los aprendices de lectura-escritura son personas que aprenden mientras leen o escriben notas. Los aprendices kinestésicos son personas que aprenden moviéndose, tocando y expresándose a través de la acción.

\section{Método}

El objetivo de un diseño eficaz del aula invertida es establecer las condiciones para un aprendizaje con atención especial a las actividades que generan la transferencia de contenidos del curso. Con el propósito de conocer el acceso a la tecnología entre los estudiantes, se realizó una encuesta, al total de la población de 22 estudiantes inscritos en el ciclo 2017. De acuerdo a la clasificación del canal perceptivo en el modelo VAK, sobre el tema de uso de Internet por tiempo, clasificación y disponibilidad de recursos tecnológicos. El $44 \%$ es visual, $30 \%$ es auditivo y $26 \%$ es kinestésico, inciso b) de la Figura 2 y sobre el tiempo dedicado a Internet el $41 \%$ de los estudiantes emplea de 4 a 8 horas diarias, inciso b) de la Figura 2.

Los estudiantes de tiempo completo no presentan inconvenientes para el acceso al entorno asíncrono del aula invertida; pero los estudiantes con un trabajo formal de ocho horas y poca disponibilidad de tiempo tienen dificultades para cumplir con las actividades asíncronas. En el inciso c) de la figura 2, se observa un porcentaje favorable a la búsqueda frecuente de información sobre temas de entretenimiento y socialización. En el inciso d) de la figura 2 se observa que el $90 \%$ de los estudiantes tiene acceso a Internet desde su casa, pero solo el $60 \%$ dispone de computadora para uso exclusivo. Un $30 \%$ cuenta con un teléfono inteligente con acceso a Internet ilimitado y la disponibilidad de una tableta electrónica es menor al 10\%. 


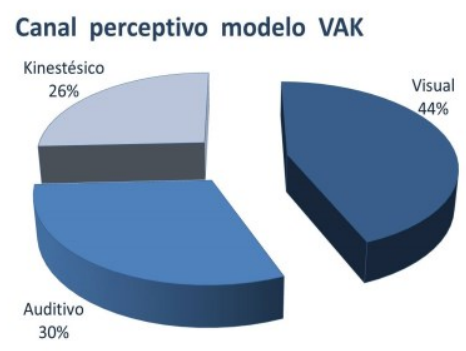

a)

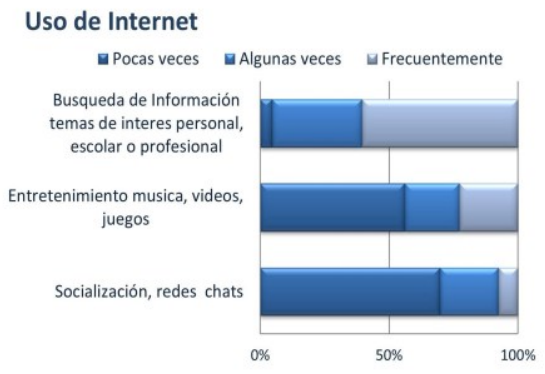

c)

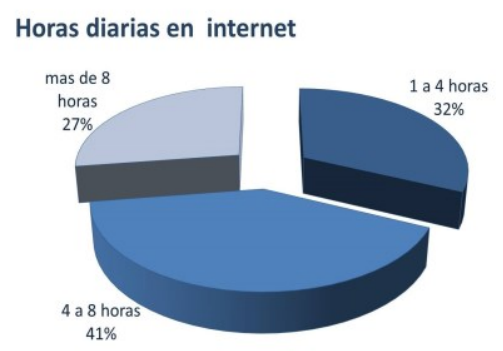

b)

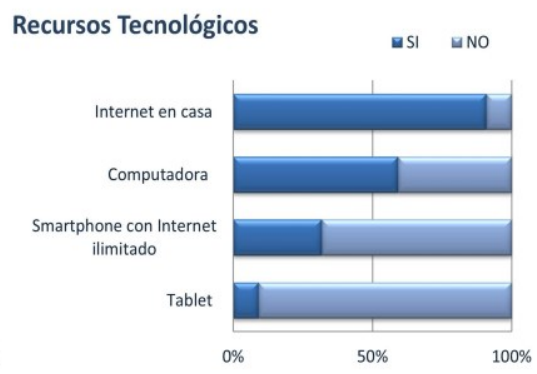

d)

Fig. 2 Resultados de la encuesta a los estudiante a) para canal perceptivo en el modelo VAK, b) uso de horas de internet por día, c) uso de internet por tipo de búsqueda y d) disponibilidad de recursos tecnologicos.

Algunos métodos y herramientas aplicados en el presente caso para evaluar el aprendizaje de los estudiantes después de la instrucción, fueron rubricas y portafolios de proyectos, porque proporcionan una forma conveniente de documentar el progreso. Además su implementación se puede llevar acabo en algunos de los sistemas de gestión del aprendizaje que incluyen aplicaciones en línea, como Moodle, Wix, Edmodo, entre otros (Estes, Ingram y Liu. 2014). En el presente trabajo la implementación fue gradual con recursos físicos durante los ciclos 2012B a 2014A, con archivos digitales enviados correo electrónico en 2014B y desde 2016A a través en un sitio de acceso libre disponible en Wix.

\section{Resultados}

Los resultados de las siguientes encuestas se clasifican en la escala de Likert, la cual ha sido utilizada en casos anteriores ( Roach, 2014) para la evaluación del modelo de aula invertida. En la Figura 3, inciso a) se muestran los resultados de la encuesta sobre competencias en TIC para comunicación y aprendizaje colaborativo, donde la mayoría menciona ser capaz de usar el correo electrónico y navegar dentro de una plataforma educativa, pero mencionan no ser capaces de participar en la creación y diseño web. 
Consideraciones para el aprendizaje autónomo en el entorno asíncrono: caso de implementación gradual de aula invertida en ingeniería.

Los resultados de la encuesta sobre competencias en TIC para búsqueda y tratamiento de la información, inciso b) de la Figura 3, los estudiantes son capaces de usar cualquier navegador, compartir videos, documentos y usar software en línea, pero carecen de habilidades para difundir información por códigos QR o de usar software especializado para administración del tiempo y síntesis de la información.

En la figura 4 se muestra la evolución de las calificaciones semestrales y la integración de elementos de aprendizaje activo en la transición de educación tradicional hacia el modelo de aula invertida en periodos semestrales, desde 2012B hasta 2016B; en la asignatura de Mecánica Clásica con estudiantes de primer ingreso de la Licenciatura de Ingeniería Electrónica, en la Unidad Académica Zapopan del Instituto Tecnológico "José Mario Molina Pasquel y Henríquez”, en el Estado de Jalisco, México.

\section{Competencias TIC para comunicación y aprendizaje colaborativo}

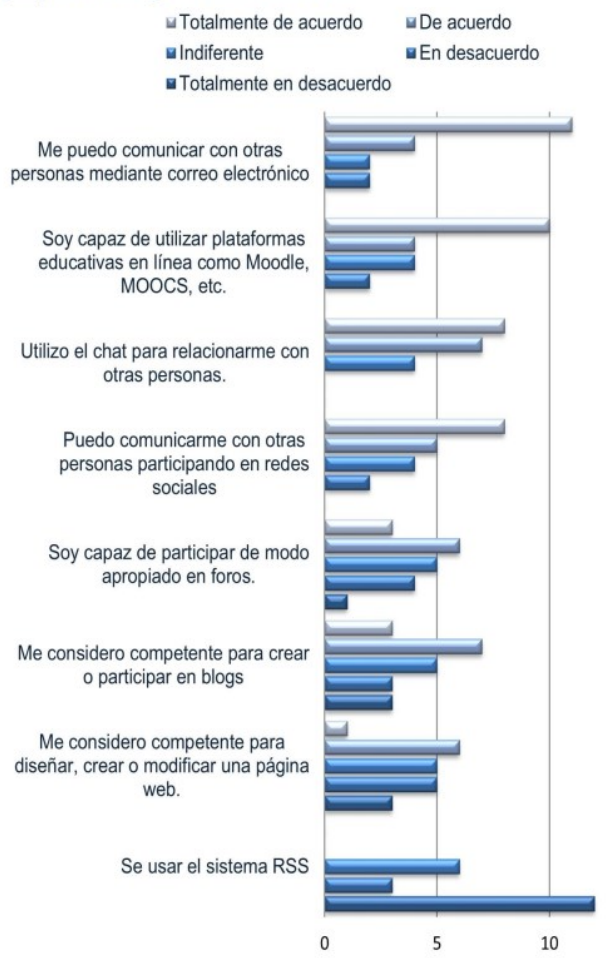

a)

\section{Competencias TIC para búsqueda y tratamiento de la información}

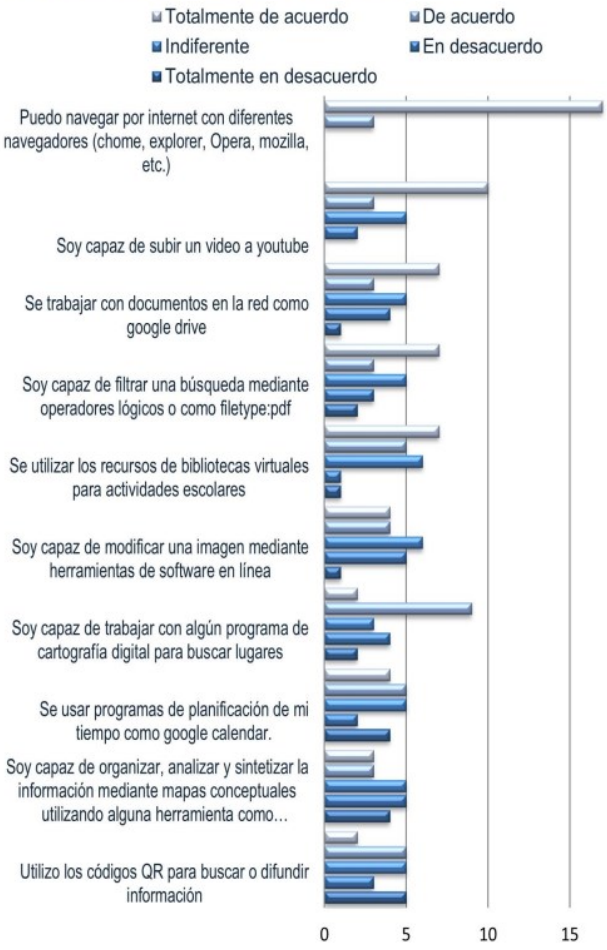

b)

Fig. 3 Resultados de las encuestas sobre competencias en TIC para: a) comunicación y aprendizaje colaborativo b) para búsqueda y tratamiento de la información 
A partir del periodo 2014A con la implementación del aprendizaje basado en problemas ABP, se mejoró el rendimiento escolar, pero en el ciclo 2016B con un mayor uso de TICS y un enfoque de aprendizaje autónomo con instrucciones y actividades asíncronas disponibles en un sitio de internet, se detectó una disminución en el rendimiento. Para revertir esta tendencia, a partir de 2017 se está trabajando en el diseño del sitio web de la materia con recursos que consideran las preferencias, canales perceptivos y competencias TIC de los estudiantes. La evaluación de estas acciones correctivas para lograr el aprendizaje autónomo en el modelo de aula invertida se propone como trabajo futuro para 2018, cuando se recaben suficientes datos. Consideramos relevante compartir con la comunidad académica el presente estudio, con la propuesta de realizar un diagnóstico de las competencias TIC de los estudiantes en la implementación del modelo de aula invertida

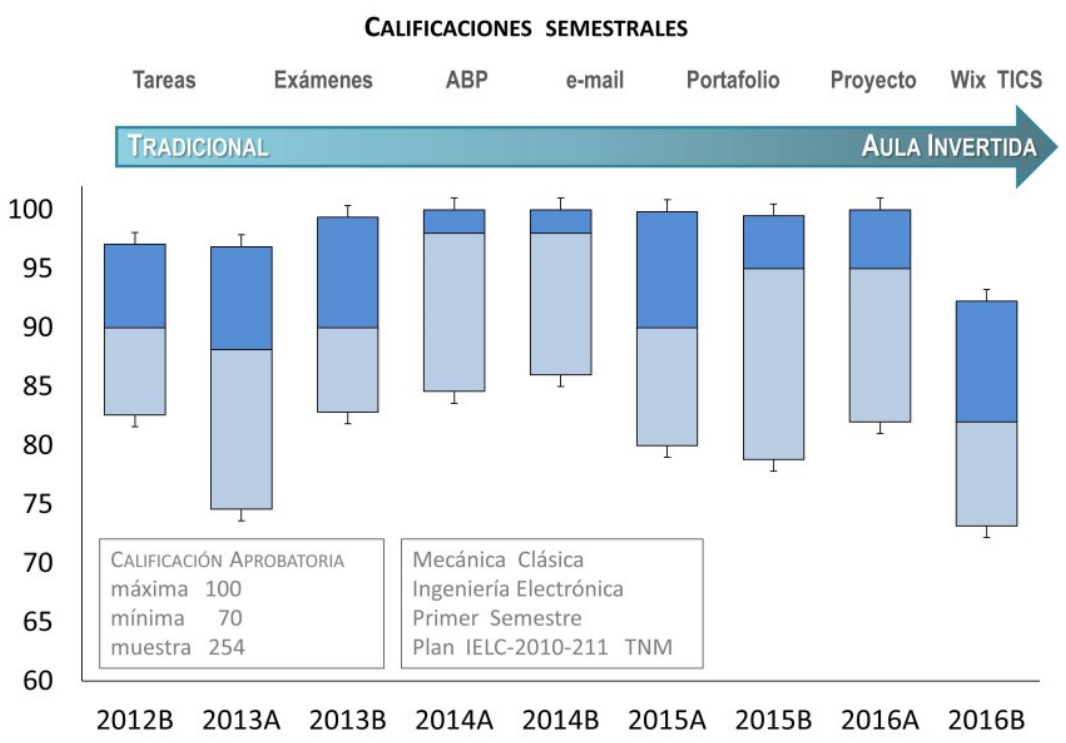

Fig. 4 Grafica de caja para calificaciones semestrales, percentiles del $25 \%$ y $75 \%$ con exclusión de calificaciones por debajo del mínimo aprobatorio. 
Consideraciones para el aprendizaje autónomo en el entorno asíncrono: caso de implementación gradual de aula invertida en ingeniería.

\section{Conclusiones}

En el entorno síncrono del aula invertida, los resultados de la implementación del aprendizaje activo basado en problemas ABP y el desarrollo de proyectos muestran un mejor rendimiento académico en los ciclos 2014A y 2016A respectivamente. Por el contrario, en el ciclo $2016 \mathrm{~B}$ con un mayor uso de TICS y un enfoque de aprendizaje autónomo con instrucciones y actividades asíncronas, se observa una disminución general en el rendimiento.

Para la ejecución de las actividades asíncronas del aula invertida, los resultados de la encuesta de diagnóstico sobre el canal perceptivo, muestran una preferencia de los estudiantes hacia recursos audiovisuales. También se concluye que la disponibilidad de tiempo y de acceso a recursos tecnológicos no es un obstáculo importante para los estudiantes del presente caso en la implementación de aula invertida.

Los resultados de las encuestas sobre competencias en TICS de los estudiantes muestran que es necesario corregir deficiencias para alcanzar el éxito del aprendizaje autónomo en el entorno asíncrono del aula invertida, en consecuencia se propone que el docente asigne un tiempo dentro del aula para el diagnóstico y el desarrollo de competencias digitales.

\section{Referencias}

Martínez Olvera, W., Esquivel Gámez, I., Martínez Castillo, J. (2014). Aula Invertida o Modelo Invertido de Aprendizaje: Origen, sustento e implicaciones. En Los Modelos Tecno-Educativos, revolucionando el aprendizaje del siglo XXI (págs. 143-160). Mexico: Editorial Lulu.

Roach, T. (2014). Student perceptions toward flipped learning: New methods to increase interaction and active learning in economics. International Review of Economics Education, 17, 74-84.

Bergmann, J., Sams, A. (2012). Flip Your Classroom: Reach Every Student in Every Class Every Day. Washington, DC: International Society for Technology in Education.

Bishop, J., Verleger, M. (June 2013). The Flipped Classroom: A Survey of the Research. 2013 ASEE Annual Conference \& Exposition (págs. 1-18). Atlanta, Georgia: https://peer.asee.org/22585

Consejo de Acreditación de la Enseñanza de la Ingeniería A.C. (2017). Marco de Referencia 2018 del CACEI en el Contexto Internacional. Recuperado de http://www.cacei.com.mx/docs/marco_ing_2018.pdf

Dahlstrom, E., Brooks, C., Grajek, S., Reeves, J. (2015). The ECAR Study of Undergraduate Students and Information Technology, 2015. Research Report. Louisville, CO: EDUCAUSE Center for Analysis and Research.

Demetry, C. (2010). Work in progress - An innovation merging "classroom flip" and team-based learning. Frontiers in Education Conference (FIE) (págs. T1E-1-T1E-2). Washington, DC: IEEE.

Estes, M., Ingram, R., \& Liu, J. (2014). A Review of Flipped Classroom Research, Practice, and Technologie. International HETL Review, 4(7). 
Flipped Learning Network. (12 de Marzo de 2014). The Four Pillars of F-L-I-PTM. Obtenido de http://flippedlearning.org/definition-of-flipped-learning/

Francl, T. (2014). Is Flipped Learning Appropriate? Journal of Research in Innovative Teaching, 7(1), 119-128.

Grabinger, R., \& Dunlap, J. (1995). Rich environments for active learning: A definition. Association for Learning Technology Journal, 3(2), 5-34.

Intel Teach Program. (2012). Learning Styles. Recuperado el 2 de Marzo de 2017, de http://www.intel.com/content/dam/www/program/education/us/en/documents/projectdesign/skills/learning-styles.pdf

Lage, M. J., Platt, G. J., Treglia, M. (2000). Inverting the Classroom: A Gateway to Creating an Inclusive Learning Environment. The Journal of Economic Education, 31(1), 30-43.

Muthyala, J. (2014). Inclusiveness with Learning Style. IJELLS, 3(1), 62-69.

Oltra Gutierrez, J.V; Estelles-Miguel, S.; Garrigos-Simón, F.J. y Montesa Andrés, J.O. (2017). Comparativa de la Experiencia de Flip Teaching en dos asignaturas y centros distintos de la UPV. Congreso IN-RED 2017. Doi: http://dx.doi.org/10.4995/INRED2017.2017.6891

Pekic, Z., Pekic, N., Kovac, D., \& Dlabac, T. (2014). How Learning styles affect the experience of ELearning. Book of Proceedings 6th International Maritime Science Conference, (págs. 106-111). Solin Croatia.

Staker, H., \& Horn, M. (May de 2012). Classifying K-12 Blended Learning. Recuperado de Innosight Institute: http://www.innosightinstitute.org/innosight/wpcontent/uploads/2012/05/Classifying-K-12-blended-learning2.pdf

Vygotsky, L., Cole, M., John-Steiner, V., Scribner, S., Souberman, E. (1980). Mind in Society Development of Higher Psychological Processes. Harvard Univ. Press.

Zhao, Y., Ho, A. (2014). Evaluating the Flipped Classroom in An Undergraduate History Course. HarvardX Research Memo. 